\title{
K-GROUPS WITH FINITE COEFFICIENTS AND ARITHMETIC
}

\author{
PAUL ARNE ØSTVAR
}

\begin{abstract}
In this paper we prove rank formulas for the even K-groups of number rings and relate Leopoldt's conjecture to K-theory. These results follow from a computation of the higher K-groups with finite coefficients.
\end{abstract}

\section{Introduction and main results}

Let $F$ be a number field. Algebraic K-theory associates to the ring of $p$-integers $\mathcal{O}_{p}$ in $F$ a sequence of groups

$$
K_{0}\left(\mathscr{O}_{p}\right), K_{1}\left(\mathscr{O}_{p}\right), K_{2}\left(\mathscr{O}_{p}\right), \ldots, K_{n}\left(\mathscr{O}_{p}\right), K_{n+1}\left(\mathscr{O}_{p}\right), \ldots
$$

called the algebraic K-groups of $\mathscr{O}_{p}$. They are the homotopy groups of a spectrum constructed from the category of finitely generated projective $\mathscr{O}_{p}$-modules [18]. It has been known for over thirty years that the first three groups in the sequence reflect arithmetic properties of $F$. See [30] for a survey. A theorem of Quillen says that these K-groups are finitely generated Abelian groups [19]. In [3], Borel computed their ranks. In this paper we consider the torsion subgroup of $K_{n}\left(\mathscr{O}_{p}\right)$. If $F$ is totally imaginary, the spectral sequence relating motivic cohomology to algebraic K-groups with finite coefficients implies the expected formulas for the mod $2^{v} \mathrm{~K}$-groups $K_{n}\left(\mathscr{O}_{2} ; \mathrm{Z} / 2^{v}\right)$.

THEOREM 1.1. Let $F$ be a number field and $n \geq 1$.

(1) If $F$ is totally imaginary, then the mod $2^{v} \mathrm{~K}$-groups of $\mathrm{O}_{2}$ are described by an isomorphism

$$
K_{2 n-1}\left(\mathscr{O}_{2} ; \mathrm{Z} / 2^{v}\right) \rightarrow H_{\text {êt }}^{1}\left(\mathscr{O}_{2} ; \mathrm{Z} / 2^{v}(n)\right)
$$

and a short exact sequence

$$
0 \rightarrow H_{\text {êt }}^{2}\left(\mathscr{O}_{2} ; \mathbf{Z} / 2^{v}(n+1)\right) \rightarrow K_{2 n}\left(\mathscr{O}_{2} ; \mathbf{Z} / 2^{\nu}\right) \rightarrow H_{\text {ét }}^{0}\left(\mathscr{O}_{2} ; \mathbf{Z} / 2^{v}(n)\right) \rightarrow 0 .
$$


(2) Assume the Bloch-Kato conjecture holds at the odd prime number $p$. Then $O_{2}$ can be replaced by $\mathcal{O}_{p}$ and $2^{v}$ by $p^{v}$ in (1) for $F$ an arbitrary number field.

The proof of Theorem 1.1 follows the argument for $v=1$ in [20]. Theorem 1.1 allows to relate the multiplication by a power of $p$ map on the Kgroups of $\mathscr{O}_{p}$ with étale cohomology.

Corollary 1.2. Assume the hypothesis in Theorem 1.1. For $n \geq 2$, there is the exact sequence

$$
\begin{array}{r}
0 \rightarrow H_{\mathrm{et}}^{0}\left(\mathscr{O}_{p} ; \mathrm{Z} / p^{v}(n)\right) \rightarrow K_{2 n-1}\left(\mathscr{O}_{p}\right) \stackrel{p^{v}}{\longrightarrow} K_{2 n-1}\left(\mathscr{O}_{p}\right) \rightarrow H_{\mathrm{et}}^{1}\left(\mathscr{O}_{p} ; \mathrm{Z} / p^{v}(n)\right) \\
\rightarrow K_{2 n-2}\left(\mathscr{O}_{p}\right) \stackrel{p^{v}}{\longrightarrow} K_{2 n-2}\left(\mathscr{O}_{p}\right) \rightarrow H_{\mathrm{et}}^{2}\left(\mathscr{O}_{p} ; \mathrm{Z} / p^{v}(n)\right) \rightarrow 0 .
\end{array}
$$

REMark. This result is known when $n=2$ by [14] and [16]. One expects to have a similar exact sequence for real number fields and the prime 2 .

Let us denote by $r_{1}$ the number of real places of $F$, and by $r_{2}$ the number of pairs of complex places of $F$. Leopoldt's conjecture predicts that the number of independent $Z_{p}$-extensions of $F$ equals $r_{2}+1$. This is known to hold for Abelian number fields, cf. Corollary 5.32 and Theorem 13.4 [28]. The étale cohomological formulation is that

$$
H_{\text {êt }}^{2}\left(\mathscr{O}_{p} ; \mathrm{Z} / p^{\infty}(0)\right)=0,
$$

or equivalently

$$
\mathrm{rk}_{\mathrm{Z}_{p}} H_{\mathrm{et}}^{1}\left(\mathcal{O}_{p} ; \mathrm{Z}_{p}(0)\right)=r_{2}+1 .
$$

The Leopoldt conjecture is part of a more general conjecture due to Schneider [21]. See [10] for a further generalization.

$\mathrm{SC}$ (for $p$ odd). The group $H_{\mathrm{et}}^{2}\left(\mathcal{O}_{p} ; \mathrm{Z} / p^{\infty}(i)\right)$ is trivial for $p$ odd and $i \neq 1$.

Théorème 5 [22] implies SC for $i \geq 2$ since $H_{\text {êt }}^{2}\left(\mathscr{O}_{p} ; \mathbf{Z} / p^{\infty}(i)\right)$ is divisible for $p$ odd. See [7] for a proof which uses étale K-theory. One expects that the case $p=2$ and $F$ totally imaginary can be included in the formulation of Schneider's conjecture, cf. Theorem 7.3 [29].

If $p=2$ and $F$ is a formally real number field, we will state a similar conjecture for the positive étale cohomology group $\mathrm{H}_{+}^{*}\left(\mathscr{O}_{2} ; \mathrm{Z} / 2^{\infty}(i)\right)$ introduced in [4]. Let $\mathrm{R}$ denote the real numbers, and consider the exact sequence

$$
\begin{aligned}
\cdots \rightarrow \oplus^{r_{1}} H_{\mathrm{et}}^{1}\left(\mathrm{R} ; \mathrm{Z} / 2^{\infty}(i)\right) \rightarrow H_{+}^{2}\left(\mathcal{O}_{2} ; \mathrm{Z} / 2^{\infty}(i)\right) \\
\rightarrow H_{\mathrm{et}}^{2}\left(\mathscr{O}_{2} ; \mathrm{Z} / 2^{\infty}(i)\right) \rightarrow \oplus^{r_{1}} H_{\mathrm{et}}^{2}\left(\mathrm{R} ; \mathrm{Z} / 2^{\infty}(i)\right) \rightarrow 0 .
\end{aligned}
$$


Here $H_{+}^{2}\left(\mathscr{O}_{2} ; \mathrm{Z} / 2^{\infty}(i)\right)$ is divisible since $H_{+}^{3}\left(\mathscr{O}_{2} ; \mathrm{Z} / 2(i)\right)=0$. Lemma 7.1.1 [29] shows that the groups appearing in the direct sums are finite. The next conjecture is therefore equivalent to $H_{\mathrm{et}}^{2}\left(\mathscr{O}_{2} ; \mathrm{Z} / 2^{\infty}(i)\right)$ being finite for $i \neq 1$.

Conjecture (for $p=2$ ). The group $H_{+}^{2}\left(\mathscr{O}_{2} ; \mathrm{Z} / 2^{\infty}(i)\right.$ ) is trivial for $i \neq 1$.

The group $H_{\text {ett }}^{2}\left(\mathcal{O}_{2} ; \mathrm{Z} / 2^{\infty}(i)\right)$ is finite for $i \geq 2$ according to Proposition 4.6 [20]. This proves the conjecture for $i \geq 2$. It seems likely that techniques from Iwasawa theory can lead to a proof of the conjecture for almost all $i$.

Theorem 1.1 gives a K-theoretic reformulation of the Schneider conjecture. Let $e_{v}$ denote the exponent of $\left(\mathrm{Z} / p^{v}\right)^{\times}$. The next result fills a minor gap in the literature on $\mathrm{K}$-groups of number fields at the prime 2 .

Proposition 1.3 (Kolster). Assume the hypothesis in Theorem 1.1 and let $i \neq 1$. The following are equivalent.

(1) SC holds for $p$ and $i$.

(2) For some $v$ and some $n \geq 2, n \equiv i \bmod e_{v}$ we have $\operatorname{rk}_{p^{v}} K_{2 n-2}\left(\mathscr{O}_{p}\right)=0$.

(3) For some $v$ and all $m, n \geq 2, m \equiv n \equiv i \bmod e_{v}$ there is an abstract isomorphism $K_{2 m-2}\left(\mathscr{O}_{p}\right) \cong K_{2 n-2}\left(\mathscr{O}_{p}\right)$ on the p-torsion part.

REMARK. See Theorem 2.3 [13] for the analogous result for étale K-groups of number fields at odd prime numbers and of non-exceptional number fields at the prime 2. Kolster's proof carries over to our situation. The approach in loc. cit. was based on the Dwyer-Friedlander spectral sequence [7]. Our approach uses the Bloch-Lichtenbaum spectral sequence with finite coefficients from [20], see also [2].

In the last part of the paper we prove a higher $p$-rank formula for the even $\mathrm{K}$-groups of $\mathscr{O}_{p}$. Let $F_{v}=F\left(\zeta_{p^{v}}\right)$ where $\zeta_{p^{v}}$ is a primitive $p^{v}$ th root of unity. Let $G_{v}$ denote the Galois group of $F_{\nu} / F$, and let $G_{\infty}$ denote the Galois group $\operatorname{Gal}\left(F_{\infty} / F\right)$ where $F_{\infty}=\bigcup F_{v}$. Denote the ring of $p$-integers in $F_{v}$ by $\mathscr{O}_{p, v}$ and write $\mathscr{O}_{p, \infty}$ for the direct limit. Define the integer $r^{(n)}(F)$ to be $r_{2}$ if $n$ is even, and $r_{1}+r_{2}$ if $n$ is odd. Write Pic for the Picard group of isomorphism classes of algebraic line bundles.

THEOREM 1.4. Assume the hypothesis in Theorem 1.1, and additionally that $F$ is non-exceptional when $p=2$. For $n \neq 1 \bmod p$, there is the $p^{v}$-rank formula

$$
\begin{aligned}
\operatorname{rk}_{p^{v}} K_{2 n-2}\left(\mathscr{O}_{p}\right)= & \operatorname{rk}_{p^{v}}\left(\mathscr{O}_{p, v}^{\times} / \mu_{p^{v}} \otimes \mathrm{Z} / p^{v}(n-1)\right)^{G_{v}} \\
& +\operatorname{rk}_{p}\left({ }_{p^{\nu}} \operatorname{Pic}\left(\mathscr{O}_{p, v}\right)(n-1)^{G_{v}} / \operatorname{im} \gamma_{n}^{v}\right)-r^{(n)}(F)
\end{aligned}
$$

where $\gamma_{n}^{v}:{ }_{p^{v-1}} \operatorname{Pic}\left(\mathscr{O}_{p, \nu-1}\right)(n-1)^{G_{v-1}} \rightarrow p^{v} \operatorname{Pic}\left(\mathscr{O}_{p, v}\right)(n-1)^{G_{\nu}}$. 
Some of the results in Section 2 were obtained in 1996 in my Master Thesis at the University of Oslo. I thank John Rognes for friendly and helpful guidance.

\section{Mod $p^{v}$ K-groups}

Denote the completion of $F$ at a prime $\wp$ by $F_{\wp}$, and by $k_{\wp}$ the residue field of $\wp$. Let $A\{p\}$ be the maximal $p$-torsion subgroup of an Abelian group $A$. From [22] and [29] there are localization sequences for étale cohomology and $\mathrm{K}$-theory groups with mod $p^{v}$-coefficients

$$
\begin{aligned}
0 \rightarrow & H_{\mathrm{ett}}^{1}\left(\mathscr{O}_{p} ; \mathrm{Z} / p^{v}(n)\right) \rightarrow H_{\mathrm{ett}}^{1}\left(F ; \mathbf{Z} / p^{v}(n)\right) \\
\rightarrow \oplus_{\wp \nmid \nmid p} & H_{\mathrm{et}}^{0}\left(k_{\wp} ; \mathrm{Z} / p^{v}(n-1)\right) \rightarrow H_{\mathrm{et}}^{2}\left(\mathscr{O}_{p} ; \mathbf{Z} / p^{v}(n)\right) \\
& \rightarrow H_{\mathrm{et}}^{2}\left(F ; \mathbf{Z} / p^{v}(n)\right) \rightarrow \oplus_{\wp \nmid \nmid p} H_{\mathrm{et}}^{1}\left(k_{\wp} ; \mathbf{Z} / p^{v}(n-1)\right) \rightarrow 0,
\end{aligned}
$$

and

$$
\begin{aligned}
0 \rightarrow K_{2 n-1}\left(\mathscr{O}_{p} ; \mathrm{Z} / p^{\nu}\right) \rightarrow K_{2 n-1}\left(F ; \mathbf{Z} / p^{\nu}\right) \\
\rightarrow \oplus_{\wp \nmid p} K_{2 n-2}\left(k_{\wp} ; \mathrm{Z} / p^{\nu}\right) \rightarrow K_{2 n-2}\left(\mathscr{O}_{p} ; \mathrm{Z} / p^{\nu}\right) \\
\rightarrow K_{2 n-2}\left(F ; \mathbf{Z} / p^{\nu}\right) \rightarrow \oplus_{\wp \nmid \nmid} K_{2 n-3}\left(k_{\wp} ; \mathrm{Z} / p^{\nu}\right) \rightarrow 0 .
\end{aligned}
$$

We will employ (2.1) and (2.2) in the calculation of $K_{n}\left(\mathscr{O}_{p} ; \mathrm{Z} / p^{\nu}\right)$.

Proof of Theorem 1.1. Let us first prove the result for the prime 2, and then comment on the conjectural part. For $p=2$ there is a mod $p^{v}$ BlochLichtenbaum spectral sequence for number fields [20]. Its $E_{2}$-page is:

$$
E_{2}^{m, n}=\left\{\begin{array}{ll}
H_{\mathrm{et}}^{m-n}\left(F ; \mathrm{Z} / 2^{v}(-n)\right) & n \leq m \leq 0 \\
0 & \text { otherwise }
\end{array} \Longrightarrow K_{-m-n}\left(F ; \mathrm{Z} / 2^{\nu}\right)\right.
$$

For $F$ a totally imaginary number field there are no non-trivial differentials for bidegree reasons. Hence the spectral sequence collapses at its $E_{2}$-page. Likewise for $F_{\wp}$. Consider now the 2-integers in $F$. The edge maps in the Bloch-Lichtenbaum spectral sequences for $F$ and $F_{\wp}$ induce the diagram (2.3)

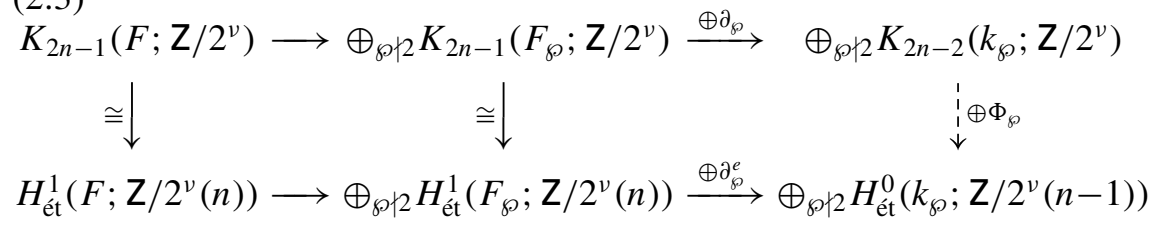

where we look for a map $\oplus \Phi_{\wp}$ that makes the diagram commute. The left hand side of (2.3) is commutative by naturality of the Bloch-Lichtenbaum 
spectral sequence with respect to the completions of $F$. The composite of the two lower horizontal maps in (2.3) equals the first connecting homomorphism in (2.1), and likewise for the two upper horizontal maps in (2.3) and the first connecting homomorphism in (2.2). Hence it suffices to find a map $\Phi_{\wp}$ that makes the diagram

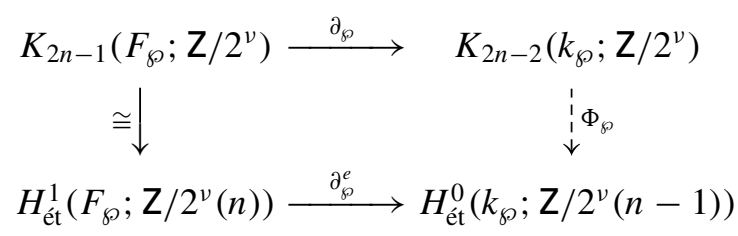

commutative (for $\wp$ non-dyadic). To that end we compare with the diagram

$$
\begin{array}{ccc}
K_{2 n-1}\left(F_{\wp} ; \mathrm{Z} / 2^{\infty}\right) \underset{\wp}{\stackrel{\partial_{\wp}}{\cong}} & K_{2 n-2}\left(k_{\wp} ; \mathrm{Z} / 2^{\infty}\right) \\
\cong & \downarrow \\
H_{\mathrm{et}}^{1}\left(F_{\wp} ; \mathrm{Z} / 2^{\infty}(n)\right) \stackrel{\partial_{\wp}^{e}}{\cong} H_{\mathrm{et}}^{0}\left(k_{\wp} ; \mathrm{Z} / 2^{\infty}(n-1)\right)
\end{array}
$$

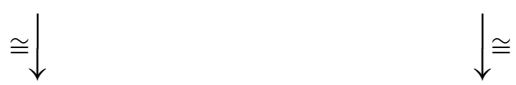

appearing in the proof of Theorem 6.3 in [20]. The existence of $\Phi_{\wp}$ follows since the map from (2.4) to the $2^{v}$-exponent subgroups in (2.5) induces an isomorphism in the upper and lower right hand corners:

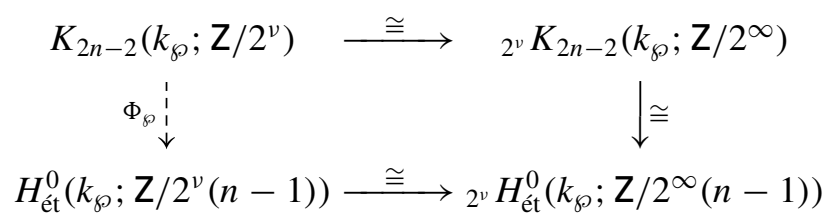

For the upper horizontal isomorphism we use the fact that $K_{2 n-1}\left(k_{\wp} ; \mathrm{Z} / 2^{\infty}\right)$ is the trivial group, and the short exact sequence

$$
0 \rightarrow K_{n+1}\left(A ; \mathbf{Z} / 2^{\infty}\right) / 2^{v} \rightarrow K_{n}\left(A ; \mathbf{Z} / 2^{\nu}\right) \rightarrow{ }_{2^{v}} K_{n}\left(A ; \mathbf{Z} / 2^{\infty}\right) \rightarrow 0
$$

for $A=k_{\wp}$. For the lower horizontal isomorphism we apply the exact sequence

$$
\begin{aligned}
0 \rightarrow H_{\mathrm{et}}^{n-1}\left(A ; \mathrm{Z} / 2^{\infty}(i)\right) / 2^{v} & \\
& \rightarrow H_{\mathrm{et}}^{n}\left(A ; \mathrm{Z} / 2^{v}(i)\right) \rightarrow 2^{v} H_{\mathrm{et}}^{n}\left(A ; \mathrm{Z} / 2^{\infty}(i)\right) \rightarrow 0 .
\end{aligned}
$$

From these remarks we note that not only does $\Phi_{\wp}$ exist, but the map is unique and in fact an isomorphism. As a result we can make the commutative 
diagram

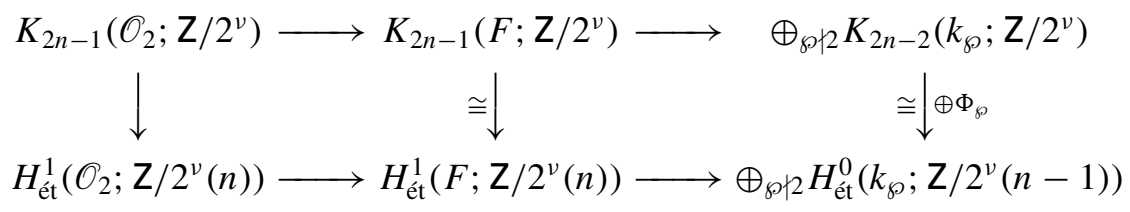

where the isomorphism in the middle is the edge map in the mod $2^{v}$ BlochLichtenbaum spectral sequence for $F$, and where the horizontal maps come from the localization sequences (2.1) and (2.2).

Consider now the even higher mod two K-groups of $\mathcal{O}_{2}$. By results in [22] and [29] we known that $H_{\mathrm{ett}}^{2}\left(\mathscr{O}_{2} ; \mathrm{Z} / 2^{\infty}(i)\right)$ is the trivial group for $i \geq 2$. From Theorem 6.3 [20] and (2.7), we have isomorphisms for $n \geq 1$ :

$$
\begin{aligned}
H_{\mathrm{et}}^{2}\left(\mathscr{O}_{2} ; \mathrm{Z} / 2^{v}(n+1)\right) & \cong H_{\mathrm{ett}}^{1}\left(\mathscr{O}_{2} ; \mathbf{Z} / 2^{\infty}(n+1)\right) / 2^{v} \\
& \cong K_{2 n+1}\left(\mathscr{O}_{2} ; \mathbf{Z} / 2^{\infty}\right) / 2^{v}
\end{aligned}
$$

Likewise, there are isomorphisms

$$
H_{\mathrm{et}}^{0}\left(\mathscr{O}_{2} ; \mathrm{Z} / 2^{v}(n)\right) \cong{ }_{2^{v}} H_{\mathrm{et}}^{0}\left(\mathscr{O}_{2} ; \mathbf{Z} / 2^{\infty}(n)\right) \cong{ }_{2^{v}} K_{2 n}\left(\mathscr{O}_{2} ; \mathbf{Z} / 2^{\infty}\right)
$$

for $n \geq 1$. The short exact sequence (2.6), (2.8) and (2.9) imply the claimed extension for $K_{2 n}\left(\mathscr{O}_{2} ; \mathrm{Z} / 2^{v}\right)$ in Theorem 1.1 (1).

Let $p$ be an odd prime number, and let $F^{\prime}$ be a field extension of $F$. The Bloch-Kato conjecture for $F^{\prime}$ at $p$ predicts that the Galois symbol

$$
K_{n}^{M}\left(F^{\prime}\right) / p^{v} \rightarrow H_{\mathrm{et}}^{n}\left(F^{\prime} ; \mathrm{Z} / p^{\nu}(n)\right)
$$

is an isomorphism. The Bloch-Lichtenbaum spectral sequence for $F$ with mod $p^{v}$-coefficients has input Voevodsky's motivic cohomology groups:

$$
E_{2}^{m, n}=H_{M}^{m-n}\left(F ; \mathrm{Z} / p^{\nu}(-n)\right) \Longrightarrow K_{-m-n}\left(F ; \mathrm{Z} / p^{\nu}\right)
$$

This depends on work in [23] and [27]. Assume the Bloch-Kato conjecture holds for every field extension of $F$. From [24] we can then identify motivic and étale cohomology of $F$ in a certain range depending on the twist. As a consequence we can rewrite the mod $p^{v}$ Bloch-Lichtenbaum spectral sequence for $F$ to have the same form as for the prime 2. The rest of the proof is then a verbatim copy of the argument given above.

Next we will use Theorem 1.1 to derive exact sequences and periodicity results for K-groups. In the following we keep the same hypothesis as in Theorem 1.1. By Theorem 6.13 [20] there is an isomorphism $H_{\text {êt }}^{0}\left(\mathscr{O}_{p} ; \mathrm{Z} / p^{\infty}(n)\right) \cong$ $K_{2 n-1}\left(\mathscr{O}_{p}\right)\{p\}$ for $p=2$. Their proof carries over to $p$ odd according to the 
discussion ending the proof of Theorem 1.1. From (2.7), there is the exact sequence

$$
0 \rightarrow H_{\mathrm{et}}^{0}\left(\mathscr{O}_{p} ; \mathrm{Z} / p^{v}(n)\right) \rightarrow K_{2 n-1}\left(\mathscr{O}_{p}\right) \stackrel{p^{v}}{\longrightarrow} K_{2 n-1}\left(\mathscr{O}_{p}\right) .
$$

By comparing the extension for $K_{2 n}\left(\mathscr{O}_{p} ; \mathrm{Z} / p^{\nu}\right)$ in Theorem 1.1 and the Bockstein exact sequence for $\mathrm{K}$-groups with finite coefficients, we find the diagram:

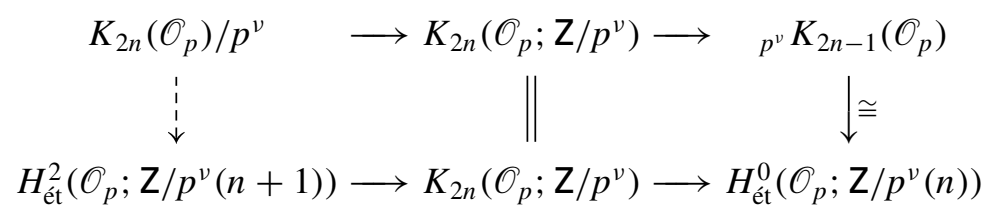

The right vertical arrow in this diagram is an isomorphism extracted from the exact sequence (2.10). It may be chosen in such a way that the diagram (2.11) commutes. This follows by comparing with a separable closure $F^{s}$ of $F$, for which there is a unique isomorphism that makes the corresponding diagram for $F^{s}$ commute. Hence, (2.11) implies exactness of the sequence

$$
K_{2 n-2}\left(\mathscr{O}_{p}\right) \stackrel{p^{v}}{\longrightarrow} K_{2 n-2}\left(\mathscr{O}_{p}\right) \rightarrow H_{\text {êt }}^{2}\left(\mathscr{O}_{p} ; \mathbf{Z} / p^{v}(n)\right) \rightarrow 0 .
$$

By applying the calculation of $K_{2 n-1}\left(\mathscr{O}_{p} ; \mathrm{Z} / p^{\nu}\right)$ to the Bockstein exact sequence, we get immediately the short exact sequence

$$
0 \rightarrow K_{2 n-1}\left(\mathscr{O}_{p}\right) / p^{v} \rightarrow H_{\mathrm{et}}^{1}\left(\mathscr{O}_{p} ; \mathrm{Z} / p^{v}(n)\right) \rightarrow{ }_{p^{v}} K_{2 n-2}\left(\mathscr{O}_{p}\right) \rightarrow 0 .
$$

Corollary 1.2 follows from (2.10), (2.12) and (2.13). From [1] we know that (2.13) is split for $p$ odd and for $v \geq 2$ if $p=2$, cf. Proposition 3.2. Next we point out some consequences of the sequences (2.10) and (2.12). If $m \equiv n \bmod e_{v}$, then we can identify $\mathbf{Z} / p^{v}(m)$ and $\mathbf{Z} / p^{v}(n)$ as coefficient sheaves in the étale topology on $\operatorname{Spec}\left(\mathscr{O}_{p}\right)$. This implies the isomorphisms

$$
\mu_{p^{v}}\left(\mathscr{O}_{p}\right) \cong{ }_{p^{v}} K_{2 e_{v}+1}\left(\mathscr{O}_{p}\right) \cong p_{p^{v}} K_{4 e_{v}+1}\left(\mathscr{O}_{p}\right) \cong \cdots
$$

and:

$$
K_{2}\left(\mathscr{O}_{p}\right) / p^{v} \cong K_{2 e_{v}+2}\left(\mathscr{O}_{p}\right) / p^{v} \cong K_{4 e_{v}+2}\left(\mathscr{O}_{p}\right) / p^{v} \cong \cdots
$$

Remark. If $F$ contains a primitive $p^{v}$ th root of unity, then $\mathrm{Z} / p^{v}(i)$ is independent of the twist $i$ and the periodicity in (2.14) and (2.15) can be decreased.

\section{Arithmetical applications}

The results in the previous section allow us to relate the Leopoldt conjecture to $\mathrm{K}$-theory, and to prove rank formulas for the even K-groups of number rings. The hypothesis in Theorem 1.1 will be assumed throughout this section. 
Proposition 1.3 implies the following result.

Corollary 3.1. If $n \geq 2$ is divisible by $e_{v}$ and $\operatorname{rk}_{p^{v}} K_{2 n-2}\left(\mathscr{O}_{p}\right)=0$, then Leopoldt's conjecture holds for $F$ at the prime number $p$.

REMARK. If the 8-rank of $K_{2}\left(\mathscr{O}_{p}\right)$ is zero and $F$ is totally imaginary, then the Leopoldt conjecture holds for $F$ at the prime 2 .

Let $w_{n}^{(p)}(F)$ denote the order of $H_{\mathrm{et}}^{0}\left(F ; \mathbf{Z} / p^{\infty}(n)\right)$ and write $\delta_{n}^{v}$ for the $p^{v}$-rank of the integers reduced modulo the greatest common divisor of $p^{v}$ and $w_{n}^{(p)}(F)$. Recall that the $p^{v}$-rank of $H_{\mathrm{et}}^{1}\left(\mathscr{O}_{p} ; \mathrm{Z} / p^{v}(0)\right)$ equals the number of independent cyclic $p$-ramified extensions of $F$ of degree $p^{v}$, cf. [9], [17] and [13]. The sequence (2.13), Borel's calculation of rational K-theory of number fields and the identification of the torsion part of $K_{2 n-1}\left(\mathscr{O}_{p}\right) \otimes Z_{p}$ from Theorem 6.13 in [20] imply the following result.

Proposition 3.2. Let $v \geq 2$ if $p=2$ and $n \geq 2$. Then:

$$
\mathrm{rk}_{p^{v}} H_{\mathrm{et}}^{1}\left(\mathscr{O}_{p} ; \mathrm{Z} / p^{v}(n)\right)=\operatorname{rk}_{p^{v}} K_{2 n-2}\left(\mathscr{O}_{p}\right)+r^{(n)}(F)+\delta_{n}^{v}
$$

In particular, the number of independent cyclic p-ramified extensions of $F$ of degree $p^{v}$ equals $\mathrm{rk}_{p^{v}} K_{2 e_{v}-2}\left(\mathscr{O}_{p}\right)+r^{(n)}(F)+1$.

REMARK. Note that the absolute Galois group of $F$ acts trivially on Z $/ 2^{v}\left(e_{v}\right)$, so $K_{2 e_{\nu}-1}\left(\mathscr{O}_{p}\right)$ contains an element of order $p^{v}$.

Let us turn to the proof of Theorem 1.4. From now on, $F$ is a non-exceptional number field if $p=2$. Then the Lyndon-Hochschild-Serre spectral sequence

$$
E_{2}^{m, n}=H^{m}\left(G_{\infty}, H_{\mathrm{et}}^{n}\left(\mathcal{O}_{p, \infty} ; \mathbf{Z} / p^{v}(i)\right)\right) \Longrightarrow H_{\mathrm{et}}^{m+n}\left(\mathscr{O}_{p} ; \mathbf{Z} / p^{v}(i)\right)
$$

collapses at its $E_{2}$-page, and there is a short exact sequence

$$
\begin{aligned}
0 \rightarrow H^{1}\left(G_{\infty}, \mathrm{Z} / p^{v}(i)\right) \rightarrow H_{\mathrm{et}}^{1}\left(\mathscr{O}_{p} ; \mathrm{Z} / p^{v}(i)\right) \\
\rightarrow H_{\mathrm{et}}^{1}\left(\mathscr{O}_{p, \infty} ; \mathrm{Z} / p^{v}(i)\right)^{G_{\infty}} \rightarrow 0 .
\end{aligned}
$$

From Tate's lemma, see p. 526 [25], there are isomorphisms for $i \neq 0$ :

$$
H_{\mathrm{et}}^{0}\left(\mathscr{O}_{p} ; \mathrm{Z} / p^{\infty}(i)\right) / p^{v} \cong H^{0}\left(G_{\infty}, \mathrm{Z} / p^{\infty}(i)\right) / p^{v} \cong H^{1}\left(G_{\infty}, \mathrm{Z} / p^{v}(i)\right)
$$

From (2.13) we get a split short exact sequence

$$
0 \rightarrow \bar{K}_{2 n-1}\left(\mathscr{O}_{p}\right) / p^{v} \rightarrow H_{\mathrm{et}}^{1}\left(\mathscr{O}_{p, \infty} ; \mathrm{Z} / p^{v}(n)\right)^{G_{\infty}} \rightarrow p^{v} K_{2 n-2}\left(\mathscr{O}_{p}\right) \rightarrow 0
$$

where $\bar{K}_{2 n-1}\left(\mathscr{O}_{p}\right)$ denotes $K_{2 n-1}\left(\mathscr{O}_{p}\right)$ modulo $p$-torsion. 
Proposition 3.4. Let $n \geq 2$. Then:

$$
\operatorname{rk}_{p^{v}} K_{2 n-2}\left(\mathscr{O}_{p}\right)=\operatorname{rk}_{p^{v}} H_{\mathrm{et}}^{1}\left(\mathscr{O}_{p, \infty} ; \mathrm{Z} / p^{v}(n)\right)^{G_{\infty}}-r^{(n)}(F)
$$

Remark. See Theorem 7.11 [20] for $r k_{2} K_{2 n-2}\left(\mathscr{O}_{2}\right)$ and any number field. Recall that $H_{\mathrm{et}}^{1}\left(\mathscr{O}_{p, \infty} ; \mathrm{Z} / p^{\infty}(n)\right)$ is isomorphic as a $\operatorname{Gal}\left(F_{\infty} / F\right)$-module to the Pontrjagin dual of the standard Iwasawa module of $F$ twisted $n$ times.

It remains to compute $\mathrm{rk}_{p^{v}} H_{\mathrm{et}}^{1}\left(\mathcal{O}_{p, \infty} ; \mathrm{Z} / p^{v}(n)\right)^{G_{\infty}}$. From Galois descent, there are isomorphisms:

$$
\begin{aligned}
H_{\mathrm{ett}}^{1}\left(\mathscr{O}_{p, \infty} ; \mathrm{Z} / p^{v}(n)\right)^{G_{\infty}} & \cong H_{\mathrm{ett}}^{1}\left(R_{F_{v}} ; \mathrm{Z} / p^{v}(n)\right)^{G_{v}} \\
& \cong H_{\mathrm{ett}}^{1}\left(R_{F_{v}} ; \mathrm{Z} / p^{v}(1)\right)(n-1)^{G_{v}}
\end{aligned}
$$

From the Kummer theory description of $H_{\mathrm{et}}^{1}\left(R_{F_{v}} ; \mathrm{Z} / p^{\nu}(1)\right)$, there is the following exact sequence

$$
\begin{aligned}
& 0 \rightarrow\left(\mathscr{O}_{p, v}^{\times} / \mu_{p^{v}} \otimes \mathrm{Z} / p^{v}(n-1)\right)^{G_{v}} \\
& \quad \rightarrow H_{\mathrm{ett}}^{1}\left(\mathscr{O}_{p, \infty} ; \mathrm{Z} / p^{v}(n)\right)^{G_{\infty}} \rightarrow{ }_{p^{v}} \operatorname{Pic}\left(R_{F_{v}}\right)(n-1)^{G_{v}} \rightarrow 0 .
\end{aligned}
$$

For exactness of (3.5) we use that $H^{1}\left(G_{v}, \mathscr{O}_{p, v}^{\times} / \mu_{p^{v}} \otimes \mathrm{Z} / p^{v}(n-1)\right)=0$ provided $n \not \equiv 1 \bmod p$, cf. Lemma 1.1 [11], p. 108 [12] or [15].

Proof of Theorem 1.4. The formula is known for $n=2$ [12]. Precisely the same arguments give the result for the higher K-groups, using the results above.

The following result is inspired by the results on relative quadratic extensions in [5], [6], [8] and [12]. Let $S_{p}$ be the set of primes of $F$ above $p$ and let $S_{p}^{d}$ be the subset of $S_{p}$ consisting of the primes which are decomposed in the extension $F / \mathrm{Q}$. We write $\# S$ for the number of elements in a finite set $S$.

Corollary 3.6. Assume the hypothesis in Theorem 1.4 and let $p$ be odd. In addition we assume that $\left[F\left(\zeta_{p^{v}}\right): F\right]=2$ and $n \not \equiv 1 \bmod p$. If $n \geq 2$ is even, then:

$$
\operatorname{rk}_{p^{v}} K_{2 n-2}\left(\mathscr{O}_{p}\right)=\# S_{p}^{d}+\operatorname{rk}_{p^{v}} \operatorname{ker}\left(\operatorname{Pic}\left(\mathscr{O}_{p, v}\right) \rightarrow \operatorname{Pic}\left(\mathscr{O}_{p}\right)\right)
$$

If $n \geq 3$ is odd, then:

$$
\operatorname{rk}_{p^{v}} K_{2 n-2}\left(\mathscr{O}_{p}\right)=\# S_{p}+\operatorname{rk}_{p^{v}} \operatorname{Pic}\left(\mathscr{O}_{p}\right)-1
$$


Proof. The group $G_{v}$ is generated by an automorphism whose restriction to the field extension $\mathrm{Q}\left(\zeta_{p^{v}}\right) / \mathrm{Q}$ is complex conjugation. Consider the surjective map:

$$
N_{F\left(\zeta_{p^{v}}\right) / F} \otimes 1: \mathcal{O}_{p, v}^{\times} / \mu_{p^{v}} \otimes \mathrm{Z} / p^{v} \rightarrow \mathscr{O}_{p}^{\times} \otimes \mathrm{Z} / p^{v}
$$

If $n \geq 2$ is even, then

$$
\left(\mathscr{O}_{p, v}^{\times} / \mu_{p^{v}} \otimes \mathrm{Z} / p^{v}(n-1)\right)^{G_{v}} \cong \operatorname{ker}\left(N_{F\left(\zeta_{p^{v}}\right) / F} \otimes 1\right)
$$

and hence $\operatorname{rk}_{p^{v}}\left(\mathscr{O}_{p, v}^{\times} / \mu_{p^{v}} \otimes \mathrm{Z} / p^{v}(n-1)\right)^{G_{v}}=\# S_{p}^{d}+r^{(n)}(F)$. Moreover, we have that:

$$
\operatorname{Pic}\left(\mathscr{O}_{p, v}\right)(n-1)^{G_{v}} \cong \operatorname{ker}\left(\operatorname{Pic}\left(\mathscr{O}_{p, v}\right) \rightarrow \operatorname{Pic}\left(\mathscr{O}_{p}\right)\right)
$$

For $n \geq 3$ odd, there are isomorphisms

$$
{ }_{p^{v}} \operatorname{Pic}\left(\mathscr{O}_{p, v}\right)(n-1)^{G_{v}} \cong{ }_{p^{v}} \operatorname{Pic}\left(\mathscr{O}_{p, v}\right)^{G_{v}} \cong{ }_{p^{v}} \operatorname{Pic}\left(\mathscr{O}_{p}\right)
$$

and:

$$
\begin{aligned}
\left(\mathscr{O}_{p, v}^{\times} / \mu_{p^{v}} \otimes \mathrm{Z} / p^{v}(n-1)\right)^{G_{v}} & \cong\left(N_{F\left(\zeta_{p} v / F\right.} \otimes 1\right)\left(\mathscr{O}_{p, v}^{\times} / \mu_{p^{v}} \otimes \mathrm{Z} / p^{v}\right) \\
& =\mathscr{O}_{p}^{\times} \otimes \mathbf{Z} / p^{v}
\end{aligned}
$$

To finish the proof we apply Theorem 1.4.

Note that the $p$-rank formulas for $K_{2 n-2}\left(\mathscr{O}_{p}\right)$ depend on the arithmetic in the tower $\cdots \subset F\left(\zeta_{p^{v}}\right) \subset F\left(\zeta_{p^{v+1}}\right) \subset \cdots$. If $\mu_{p^{v}} \subset F$, Kummer theory and the sequence (2.13) imply the following split short exact sequence

$$
0 \rightarrow K_{2 n-1}\left(\mathscr{O}_{p}\right) / p^{v} \rightarrow \mu_{p^{v}} \otimes \Delta_{F} \rightarrow p^{v} K_{2 n-2}\left(\mathscr{O}_{p}\right) \rightarrow 0 .
$$

Here $\Delta_{F}=\left\{[z] \in F^{\times} / p^{v} \mid v_{\wp}(z) \equiv 0 \bmod p^{v}\right.$ for all $\left.\wp \notin S_{p}\right\}$ and there is a split short exact sequence

$$
0 \rightarrow \mu_{p^{v}} \otimes \mathscr{O}_{p}^{\times} \rightarrow \mu_{p^{v}} \otimes \Delta_{F} \rightarrow \mu_{p^{v}} \otimes \operatorname{Pic}\left(\mathscr{O}_{p}\right) \rightarrow 0 .
$$

For the following result, see Theorem 6.2 [26] when $n=2$.

Corollary 3.9. If $\mu_{p^{v}} \subset F$ and $n \geq 2$, then:

$$
\operatorname{rk}_{p^{v}} K_{2 n-2}\left(\mathscr{O}_{p}\right)=\operatorname{rk}_{p^{v}} \operatorname{Pic}\left(\mathscr{O}_{p}\right)+r_{1}+r_{2}-r^{(n)}(F)+\# S_{p}-1
$$

Proof. This follows from (3.7) and (3.8). 


\section{REFERENCES}

1. Araki, S. and Toda, H., Multiplicative structure in mod q cohomology theories, I and II, Osaka J. Math. 2, 3 (1965, 1966), 71-115, 80-120.

2. Bloch, S. and Lichtenbaum, S., A spectral sequence for motivic cohomology, UIUC $K$-Theory preprint server, 1995.

3. Borel, A., Stable real cohomology of arithmetic groups, Ann. Sci. École Norm. Sup. 7 (1974), 235-272.

4. Chinburg, T., Kolster, M., Pappas, G. and Snaith, V., Galois structure of K-groups of rings of integers, K-Theory 14 (1998), 319-369.

5. Conner, P. E. and Hurrelbrink, J., Class number parity, Series Pure Math. 8, World Scientific Publ. Co., Singapore, 1986.

6. Conner, P. E. and Hurrelbrink, J., The 4-rank of $K_{2}(\mathcal{O})$, Canad. J. Math. XLI (1989), 932-690.

7. Dwyer, W. G. and Friedlander, E. M., Algebraic and étale K-theory, Trans. Amer. Math. Soc. 292 (1985), 247-280.

8. Hurrelbrink, J. and Kolster, M., Tame kernels under relative quadratic extensions and Hilbert symbols, J. Reine Angew. Math. 499 (1998), 145-188.

9. Iwasawa, K., On $\mathbf{Z}_{l}$-extensions of algebraic number fields, Ann. of Math. 98 (1973), 246-326.

10. Jannsen, U., On the l-adic cohomology of varieties over number fields and its Galois cohomology, Galois groups over Q (Y. Ihara, K. Ribet and J.-P. Serre, eds.), Math. Sci. Res. Inst. Publ., vol. 16, 1989, 315-360.

11. Kolster, M., On torsion in $K_{2}$ of fields, J. Pure Appl. Algebra 74 (1991), 257-273.

12. Kolster, M., $K_{2}$ of rings of algebraic integers, J. Number Theory 42 (1992), 103-122.

13. Kolster, M., Remarks on étale K-theory and the Leopoldt's conjecture, Séminaire de Théorie des Nombres, Paris, 1991-1992, Progress in Math., vol. 116, 1993, 37-62.

14. Levine, M., The indecomposable $K_{3}$ of fields, Ann. Scient. Éc. Norm. Sup. 22 (1989), 255344.

15. Merkurjev, A. S., Torsion in $K_{2}$ groups of fields, Mathematica (Leningrad University) 21 (1988), 17-20.

16. Merkurjev, A. S. and Suslin, A. A., The group $K_{3}$ for a field, Math. USSR-Izv. 36 (1991), 541-565.

17. Nguyen Quang Do, T., Sur la $\mathrm{Z}_{p}$-torsion de certains modules Galoisiens, Ann. Inst. Fourier (Grenoble) 36 (1986), 27-46.

18. Nguyen Quang Do, T., Higher algebraic K-theory, I, Lecture Notes in Math. (H. Bass, ed.), vol. 341, 1973, 85-147.

19. Nguyen Quang Do, T., Finite generation of the groups $K_{i}$ of rings of algebraic integers, Lecture Notes in Math. (H. Bass, ed.), vol. 341, 1973, 179-210.

20. Rognes, J. and Weibel, C. A., Two-primary algebraic $K$-theory of rings of integers in number fields, J. Amer. Math. Soc. 13 (2000), 1-54.

21. Schneider, P., Über gewisse Galoiscohomologiegruppen, Math. Z. 168 (1979), 181-205.

22. Soulé, C., K-théorie des anneaux d'entiers de corps de nombres et cohomologie étale, Invent. Math. 55 (1979), 251-295.

23. Suslin, A. A., Higher Chow groups and étale cohomology, Cycles, transfers, and motivic homology theories, Ann. of Math. Stud., vol. 143, 2000.

24. Suslin, A. A. and Voevodsky, V., The Bloch-Kato conjecture and motivic cohomology with finite coefficients, The arithmetic and geometry of algebraic cycles (B. B. Gordon, J. D. Lewis, S. Müller-Stach, S. Saito and N. Yui, eds.), NATO ASI Series C, vol. 548, Kluwer, 2000, 117-189.

25. Tate, J., Letter from Tate to Iwasawa on a relation between $K_{2}$ and Galois cohomology, Lecture Notes in Math. (H. Bass, ed.), vol. 342, 1973, 524-527.

26. Tate, J., Relations between $K_{2}$ and Galois cohomology, Invent. Math. 36 (1976), 257-274. 
27. Voevodsky, V., Triangulated categories of motives over a field, Cycles, transfers, and motivic homology theories, Ann. of Math. Stud., vol. 143, 2000.

28. Washington, L. C., Introduction to Cyclotomic Fields, $2 n d$ Ed., Graduate Texts in Mathematics, vol. 83, 1997.

29. Weibel, C. A., Étale Chern classes at the prime 2, Algebraic $K$-theory and Algebraic Topology (P. Goerss and J. F. Jardine, eds.), NATO ASI Series C, vol. 407, Kluwer, 1993, 249-286.

30. Weibel, C. A., The development of algebraic K-theory before 1980, Algebra, K-theory, groups, and education, Contemp. Math., vol. 243, 1999, 211-238.

DEPARTMENT OF MATHEMATICS

NORWEGIAN UNIVERSITY OF SCIENCE

AND TECHNOLOGY

TRONDHEIM

NORWAY

E-mail: ostvar@math.ntnu.no 